\title{
Sensitivity of a long-range numerical weather forecast model to small changes of model parameters
}

\author{
M. B. Gavrilov ${ }^{1}$, G. R. Jovanović ${ }^{2}$, and Z. Janjićc $\hat{c}^{3}$ \\ ${ }^{1}$ Chair of Physical Geography, Faculty of Sciences, University of Novi Sad, \\ Trg D. Obradovića 3, 21000 Novi Sad, Serbia \\ ${ }^{2}$ Hydrometeorological Service of Serbia, Kneza Višeslava 66, 11000 Belgrade, Serbia \\ ${ }^{3}$ National Centres for Environmental Predictions, Washington, USA
}

Received: 20 September 2010 - Revised: 3 January 2011 - Accepted: 11 January 2011 - Published: 1 February 2011

\begin{abstract}
Sensitivity of extended-range numerical weather forecasts to small changes of model parameters is studied for two cases. In the first case the Earth radius was perturbed. In the other case changes of the gravity were introduced. The results for the $500 \mathrm{hPa}$ geopotential fields are presented on hemispheric maps and intercompared visually and using RMS differences of the perturbed and reference forecasts. During about the first 10 days of integration the results indicate modest sensitivity of the forecasts to the parameter variation. After this period the forecasts diverge rapidly and start to differ significantly. Repeated integrations on the same computer using the same model setup and the same initial conditions yield identical results.
\end{abstract}

\section{Introduction}

Predictability of the atmosphere and accuracy of atmospheric models are still among the central issues of meteorology and atmospheric modeling. Thompson (1957) was among the first to investigate the limits of predictability of the barotropic and baroclinic atmospheres. Lorenz (1969a, b, 1973) continued and expanded this research. These investigations showed that the limit of predictability for an atmospheric model was about 12 days. The sensitivity tests of numerical integrations to small changes in initial conditions in the works of Lorenz $(1963,1965)$ were not only important for testing the accuracy and predictability of atmospheric models, but also led to the discovery of a new phenomenon in nonlinear systems known as deterministic chaos. The problem of sensitivity of numerical integrations to small disturbances of initial conditions was addressed in more recent papers, e.g., by Zhao-Xia et al. (1997) and Yoden (2007).

In the present paper, we keep the initial conditions unchanged, and instead vary within the respective limits of their natural variability two model parameters that are commonly assumed to be constant. The two parameters chosen for testing are the Earth radius and gravity. By doing so we wanted to demonstrate that the growth of differences between the so-

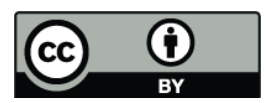

Correspondence to: M. B. Gavrilov (gavrilov@eunet.rs) lutions can be influenced not only by uncertainties in the initial conditions, but also by other small uncertainties present in the system. The specific mechanisms of the growth of the differences may be similar in both cases, though

\section{The model}

The new NCEP's Nonhydrostatic Multiscale Model on the B grid (NMMB) (Janjić, 2005; Janjić and Black, 2007; Janjić et al., 2011) was used in the tests. The NMMB was designed for a broad range of spatial and temporal scales and follows the general modeling philosophy of the NCEP's WRF NMM grid-point dynamical core (Janjić et al., 2001; Janjić, 2003,; Janjić et al., 2010). However, in contrast to the WRF NMM that was defined on the Arakawa E grid, the NMMB was reformulated for the Arakawa B grid. The NMMB uses the regular latitude-longitude grid for the global domain. Finitevolume horizontal differencing is employed that conserves a variety of basic and derived dynamical and quadratic quantities and preserves some important properties of differential operators. The conservation of energy and enstrophy (Janjić, 1984) improves the accuracy of the nonlinear dynamics of the model on all scales, and renders the model suitable for extended integrations. Conservative polar boundary conditions are used in global integrations. The polar filter selectively reduces tendencies and thus decelerates the wave components that would otherwise propagate faster in the zonal direction 
than the fastest wave propagating in the meridional direction. In the vertical, the hybrid pressure-sigma coordinate is used (Simmons and Burridge, 1981; Eckerman, 2009). Explicit, no split time differencing is applied in the model dynamics (Janjić, 2003). An affordable conservative, positive definite and monotone scheme was developed for model tracers (Janjić et al., 2009). The model physics is an evolved version of the NCEP's standard WRF NMM physical package. It includes land surface processes (Vuković et al., 2010), turbulence (Janjić, 1990, 2001), microphysics (Ferrier et al., 2002), moist convection (Janjić, 1994, 2000) and radiation (Harshvardhan et al., 1987).

\section{Experiment setup}

Two sensitivity tests of extended-range numerical simulations to small changes of Earth radius and gravity were made. The experiments were performed using reduced reference value of the Earth radius $a=6376000 \mathrm{~m}$ by $0.000002 \%$, which resulted in $a=6375999 \mathrm{~m}$, and by reducing the reference value by $0.5 \%$, which yielded $a=6344000 \mathrm{~m}$ (Gavrilov, 2006). In the spherical approximation used in the atmospheric equations the Earth radius is assumed to be a constant (e.g. $a=6376000 \mathrm{~m}$ ), although the Earth's shape is a geoid with the radius at the equator being $a=6378000 \mathrm{~m}$ and at the poles being $a=6357000 \mathrm{~m}$. Moreover, the shallow approximation ignores the radius change with height. Instead of varying between the bottom and the top of the atmosphere, that makes a distance of about $30000 \mathrm{~m}$ in atmospheric models, with this approximation the radius is taken to be constant and equal to its value at the sea level (Phillips, 1966). It is therefore evident that the variation of the Earth radius used in the experiments remains within the range of natural variability of the Earth radius. Thus, the perturbations used here have a realistic scale.

In the second set of experiments, the reference value of the gravity $g=9.8060226 \mathrm{~ms}^{-2}$ was increased by $0.0001 \%$ of the reference value to $g=9.8060326 \mathrm{~ms}^{-2}$, and reduced by $0.1 \%$ of the reference value to $g=9.7960226 \mathrm{~ms}^{-2}$ (Gavrilov et al., 2007). In the atmospheric models, the gravity is mostly taken to be constant (e.g. $g=9.8060226 \mathrm{~ms}^{-2}$ ) although it is well known that it is a function of latitude, altitude and inhomogeneity of the Earth's interior. For instance, because of changes of centrifugal force along the latitude and flattened shape of the Earth at the poles, the gravity is $0.5 \%$ higher at the poles than at the equator. Also, as the altitude is increasing, the gravity is decreasing, so that at the altitude of $1000 \mathrm{~m}$ above the sea level it is less than at the sea level by $0.00389 \mathrm{~ms}^{-2}$. Beside these theoretically predictable changes of gravity, there are gravitational anomalies occurring as the effect of inhomogeneity of the inner layers of the Earth. For instance, in North Pacific close to Hawaii, the gravity is for $0.002 \mathrm{~ms}^{-2}$ greater than the reference value. Thus, it is evident that the variation of gravity in our esperiments and the natural variability of gravity are of the same order of magnitude.

In the tests the model integrations were performed up to 40 days. The horizontal resolution was about 0.67 degrees and there were 32 levels in the vertical. The time step was $160 \mathrm{~s}$.

\section{Results}

The results shown were obtained from numerical integrations starting from the NCEP's GFS analysis for 4 February 2006, at 00:00 UTC. In Fig. 1 the $500 \mathrm{hPa}$ geopotential fields at days 10 and 14 are presented, respectively. The results of the reference run and the two runs with perturbud values of the Earth radius are shown in the top to bottom panels in the figures. The results with perturbed gravity are presented in Fig. 2 using the same arrangement. The RMS differences of the geopotential fields at $500 \mathrm{hPa}$ between the perturbed and reference runs are computed at days 5, 10, 14, 20, 30 and 40 and shown in Fig. 3 for the perturbations of the Earth's radius and gravity, respectively.

As can be seen in Figs. 1 and 2 (left columns), during the first 10 days of integration the forecasts remain generally similar. At the 5th day the RMS differences were less than $2 \mathrm{gpm}$ for both tested parameters, while at the 10th day the RMS difference was about $12 \mathrm{gpm}$ for the gravity perturbation case, and 18.5-20.7 gpm for the Earth radius perturbation case. There was no indication of regularity in the appearance of the differences and they did not show clear dependence on which parameter is perturbed and for how much.

Between the 11th and 13th day of integration a rapid degradation of previously existing similarity occurred in all cases. As shown in Figs. 1 and 2 (right columns), at day 14 the differences between the reference fields and the fields obtained using pertubed parameters are no longer small and the fields do not resemble each other any more. The differences are such that one cannot detect any indication that the integrations started from the same initial conditions. The similarity between the forecasts has never been regained in further integrations up to 40 days. As can be seen from Fig. 3, the increasing visual differences in forecast fields are confirmed by significant increase of RMS differences at days 14, 20, 30 and 40. These RMS differences take on values from $45 \mathrm{gpm}$ to $95 \mathrm{gpm}$, which are comparable to typical RMS errors for forecasts of 5-10 days (Shukla, 2005).

Analogous results were obtained in integrations starting from NCEP's GFS analyses for the whole period 4-14 February 2006 at 00:00 and 12:00 UTC. Comparable results were obtained also for other values of the Earth radius and gravity within the range of the changes discussed here. However, repeated integrations on the same computer and using the same values of model parameters gave identical final results. 

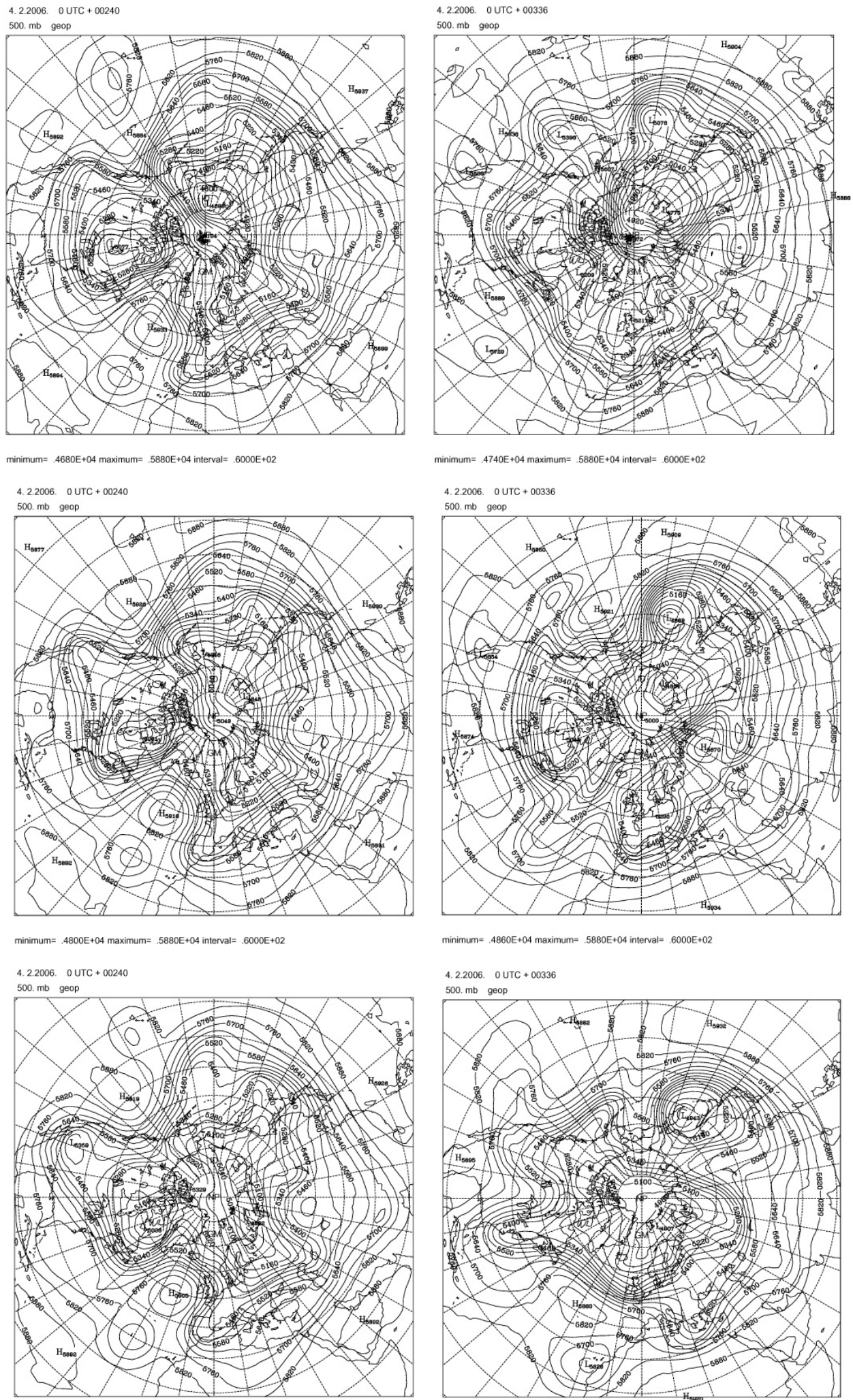

minimum=.4860E +04 maximum $=.5880 \mathrm{E}+04$ interval $=.6000 \mathrm{E}+02$

minimum $=.4860 \mathrm{E}+04$ maximum $=.5880 \mathrm{E}+04$ interval= $.6000 \mathrm{E}+02$

Figure 1. 10-days forecasts (left) and 14-days forecasts (right) of geopotential at $500 \mathrm{hPa}$ for $a=6376000 \mathrm{~m}$ (top), $a=6375999 \mathrm{~m}$ (middle) and $a=6344000 \mathrm{~m}$ (bottom). 

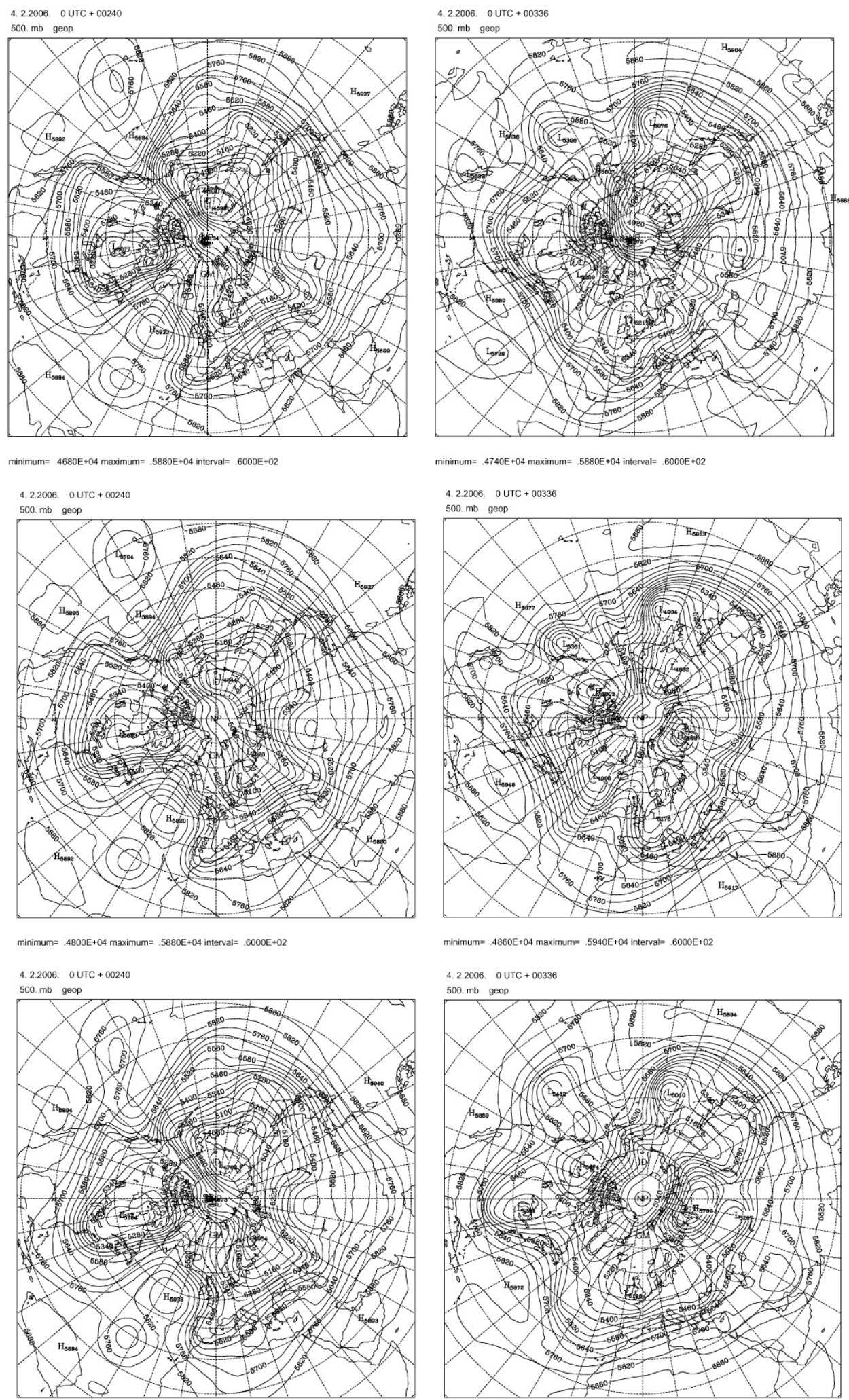

nimum $=.4620 \mathrm{E}+04$ maximum $=.5880 \mathrm{E}+04$ interval= $=.6000 \mathrm{E}+02$

minimum $=.4920 \mathrm{E}+04$ maximum $=.5880 \mathrm{E}+04$ interval $=.6000 \mathrm{E}+02$

Figure 2. 10-day forecasts (left) and 14-days forecasts (right) of $500 \mathrm{hPa}$ geopotential for $g=9.8060226 \mathrm{~ms}^{-2}$ (top), $g=9.8060326 \mathrm{~ms}^{-2}$ (middle) and $g=9.7960226 \mathrm{~ms}^{-2}$ (bottom). 

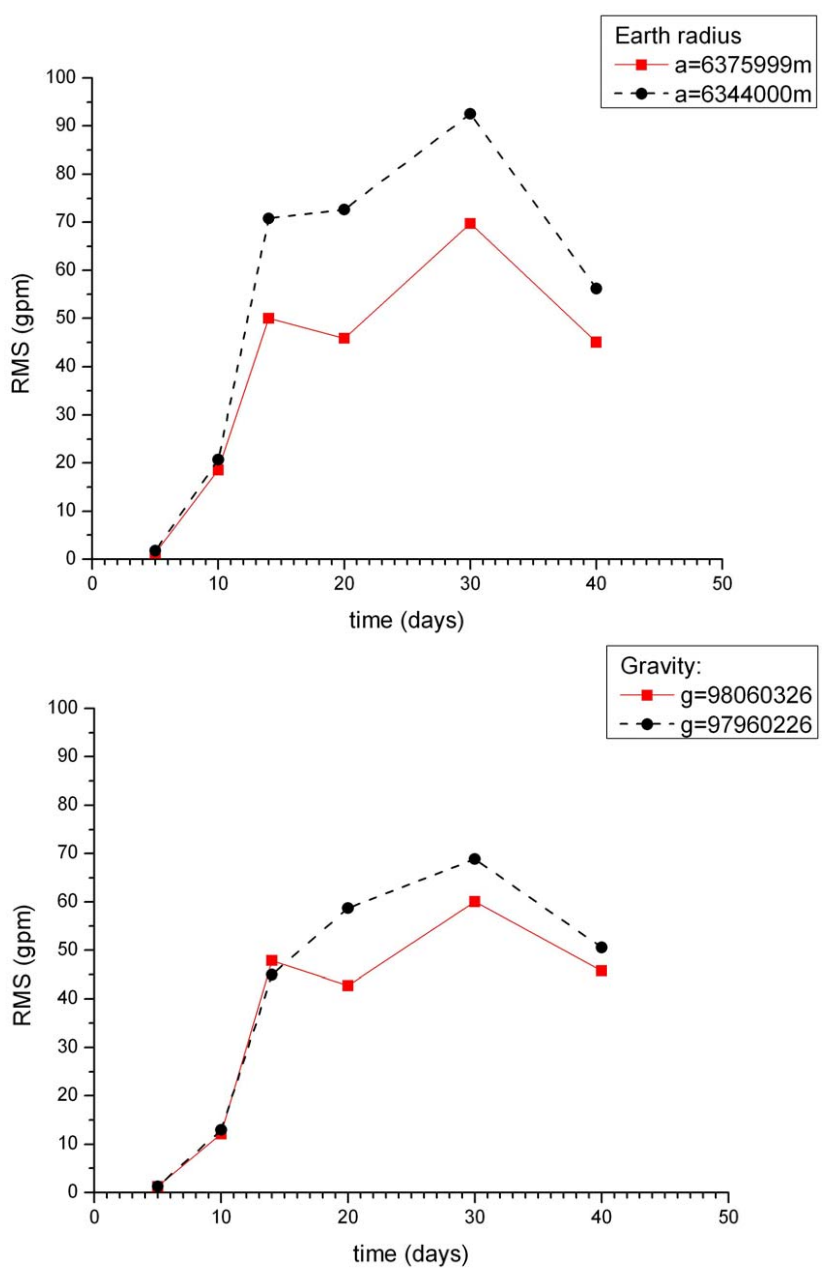

Figure 3. RMS differences for small changes of Earth radius (top) and gravity (bottom) as indicated in the legend.

\section{Summary and conclusions}

The sensitivity has been investigated of extended-range numerical simulations to small perturbations of Earth radius and gravity within the limits of their natural variabilities. Regardless of the value used for the Earth radius and gravity, the weather patterns have kept mutual visual similarity during the first 10 days. High similarity of the fields is confirmed by low values of their RMS differences. Within this period, the forecasts still gave an impression that they all started from the same initial conditions.

After this period of similarity, the results started to differ. Between the 11th and the 13th day, the differences became significant even on large scales, and the similarity never reappeared again in further integrations. High diversity of the fields is confirmed by large values of their RMS differences. In these later stages the predicted flow patterns give an impression that the forecasts were made starting from different initial conditions.
Since the results of numerical weather prediction have not shown considerable sensitivity to the small changes of model parameters in the first 10 days, these forecasts may be considered to fall in the predictable range and may have practical value. Contrary to that, great sensitivity of the deterministic weather forecasts to the small changes beyond day 10 makes the possibility of practical use of such forecasts highly questionable.

The obtained results appear to be in accord with the theoretical assessment that the deterministic predictability of the atmosphere is limited to about 12 days (Lorenz, 1973). However, the results of this study show that the growth of differences between the solutions can be influenced not only by uncertainties in the initial conditions, but also by other small uncertainties present in the system, although the specific mechanisms of the growth of the differences may be similar in both cases. It would be interesting to further investigate the influence of small changes of other model parameters (e.g., the angular velocity of the Earth and various physical and numerical constants) on the results of deterministic medium-range numerical weather forecasts.

Acknowledgements. This work was supported by Project \#176020 of the Serbian Ministry of Science and Technological Development. The authors sincerely appreciate the efforts of Igor Pisnichenko and another two anonymous reviewers in improving this manuscript.

Edited by: B. Lalic

Reviewed by: I. A. Pisnichenko, H. Scheifinger, and another anonymous referee

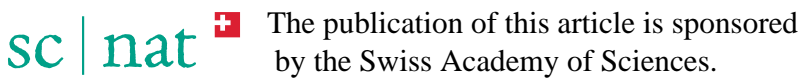

\section{References}

Eckermann, S.: Hybrid $\sigma$-p coordinate choices for a global model, Mon. Wea. Rev., 137, 224-245, 2009.

Ferrier, S. B., Jin, Y., Lin, Y., Black, T., Rogers, E., and DiMego, G.: Implementation of a new grid-scale cloud and precipitation scheme in the NCEP Eta model, 19th Conf. on Weather Analysis and Forecasting/15th Conf. on Numerical Weather Prediction, San Antonio, TX, Amer. Meteor. Soc., 280-283, 2002.

Gavrilov, M. B.: Sensitivity of Long Range Numerical Weather Prediction on Small Change of Initial Condition, Physica Macedonica - J. Exp. Theo. Phys., 56, 23-28, 2006.

Gavrilov, M., Janjić, Z., and Jovanovic, G.: The sensitivity of a long numerical weather forecasting model, 8th European Conf. on Applications of Meteorology (ECAM), San Lorenco de El Escorial, Spain, 1-5 October 2007, Abstr. EMS2007-A-00586, 2007.

Harshvardhan, R. D., Randall, D. A., and Corsetti, T. G.: A fast radiation parameterization for general circulation models, J. Geophys. Res., 92, 1009-1016, 1987. 
Janjić, Z. I.: Non-linear advection schemes and energy cascade on semi-staggered grids, Mon. Wea. Rev., 112, 1234-1245, 1984.

Janjić, Z. I.: The step-mountain coordinate: physical package, Mon. Wea. Rev., 118, 1429-1443, 1990.

Janjić, Z. I.: The step-mountain eta coordinate model: further developments of the convection, viscous sublayer and turbulence closure schemes, Mon. Wea. Rev., 122, 927-945, 1994.

Janjić, Z. I.: Comments on "Development and Evaluation of a Convection Scheme for Use in Climate Models", J. Atmos. Sci., 57, p. 3686, 2000.

Janjić, Z. I.: Nonsingular Implementation of the MellorYamada Level 2.5 Scheme in the NCEP Meso model, NOAA/NWS/NCEP Office Note \#437, p. 61, 2001.

Janjić, Z. I.: A Nonhydrostatic Model Based on a New Approach, Meteor. Atmos. Phys., 82, 271-285, 2003.

Janjić, Z. I., Gerrity, J. P., and Nickovic, S.: An Alternative Approach to Nonhydrostatic Modeling, Mon. Wea. Rev., 129, 1164-1178, 2001.

Janjić, Z. I.: A unified model approach from meso to global scales, Geophys. Res. Abstr., 7, 05582, 2005, European Geosciences Union, General Assembly, Vienna, Austria, 24-29 April 2005.

Janjić, Z. I. and Black, T.: An ESMF unified model for a broad range of spatial and temporal scales, Geophys. Res. Abstr., 9, 05025, 2007, European Geosciences Union, General Assembly, Vienna, Austria, 15-20 April 2007.

Janjić, Z., Huang, H., and Lu, S.: A unified atmospheric model suitable for studying transport of mineral aerosols from meso to global scales, IOP/Electronic J., Conf. Ser.: Earth and Envir. Sci. (collection of papers from WMO/GEO Expert Meet. on an Inter. Sand and Dust Storm Warning System Meeting, Barcelona, Spain), 9, 6, 7-9 November 2009.

Janjić, Z., Gall, R., and Pyle M. E.: Scientific Documentation for the NMM Solver, NCAR Tech. N. NCAR/TN-477+STR, 54, 2010.

Janjić, Z., Janjić, T., and Vasic, R.: A class of conservative fourth order advection schemes and impact of enhanced formal accuracy on extended range forecasts, Mon. Wea. Rev., doi:10.1175/2010MWR3448.1, in press, 2011.
Lorenz, E. N.: Deterministic nonperiodic flow, J. Atmos. Sci., 20, 130-141, 1963.

Lorenz, E. N.: A study of the predictability of a 28 variable atmospheric model, Tellus, 17, 321-333, 1965.

Lorenz, E. N.: Atmospeheric Predictability as Revealed by Naturally Occurring Analogues, J. Atmos. Sci., 26, 636-646, 1969a.

Lorenz, E. N.: The predictability of a flow which proccesse many scales of motions, Tellus, 21, 289-307, 1969b.

Lorenz, E. N.: On the Existence of Extended Range Predictability, J. Appl. Meteor., 12, 543-546, 1973.

NCEP: www.ncep.noaa.gov, ftp://ftpprd.ncep.noaa.gov/pub/data/ nccf/com/gfs/prod/, 2006.

Phillips, N. A.: The equations of motions for a shallow rotating atmosphere and the traditional approximation, J. Atmos. Sci., 23, p. 626, 1966.

Shukla, J.: Have We Reached the Limit of Weather Predictability? Lorenz Symposium, Amer. Meteor. Soc. Ann. Meet., San Diego, CA, 13 January 2005.

Simmons, A. J. and Burridge, D. M.: An energy and angularmomentum conserving vertical finite-difference scheme and hybrid vertical coordinates, Mon. Wea. Rev., 109, 758-766, 1981.

Thompson, P. D.: Uncertainty of Initial State as a Factor in the Predictability of Large Scale Atmospheric Flow Patterns, Tellus, 9, 275-295, 1957.

Vuković, A., Rajković, B., and Janjić, Z.: Land Ice Sea Surface Model: Short Description and Verification, Proceedings, paper S.02.08, 5th Inter. Con. on Environmental Modelling and Software, Ottawa, Ontario, Canada, 5-8 July 2010.

Yoden, S.: Atmospheric Predictability, J. Meteor. Soc., Japan, 85B, 77-102, 2007.

Zhao-Xia, P., Kalnay, E., Sela, J., and Szunyogh, I.: Sensitivity of forecast errors to initial conditions witha quasi-inverse linear method, Mon. Wea. Rev., 125, 2479-2503, 1997. 